\title{
Four Week Hypofractionated Accelerated Intensity Modulated Radiotherapy and Synchronous Carboplatin or Cetuximab in Biologically Staged Oropharyngeal Carcinoma
}

\author{
H. Benghiat ${ }^{1}$, P. Sanghera ${ }^{1,2}$, J. Cashmore ${ }^{1}$, J. Hodson ${ }^{3}$, H. Mehanna ${ }^{2}$, R. Simmons ${ }^{1}$, P. Massey ${ }^{1}$, G. Sangha ${ }^{1}$, \\ C. Bode ${ }^{1}$, P. Cooper ${ }^{1}$, J. Glaholm ${ }^{1} \&$ A. Hartley ${ }^{1,2}$ \\ ${ }^{1}$ Hall-Edwards Radiotherapy Research Group, Cancer Centre, Queen Elizabeth Hospital, Birmingham, UK \\ ${ }^{2}$ Institute of Head and Neck Studies and Education (InHANSE), University of Birmingham, Birmingham, UK \\ ${ }^{3}$ Wolfson Computer Laboratory, Queen Elizabeth Hospital, Birmingham, UK \\ Correspondence: H. Benghiat, Hall-Edwards Radiotherapy Research Group, Cancer Centre, Queen Elizabeth \\ Hospital, Birmingham, UK. Tel: 44-121-371-2000. E-mail: helen.benghiat@uhb.nhs.uk
}

Received: May 22, 2014

Accepted: June 16, $2014 \quad$ Online Published: June 25, 2014

doi: $10.5539 / \operatorname{cco} . v 3 n 2 p 1$

URL: http://dx.doi.org/10.5539/cco.v3n2p1

\begin{abstract}
Hypofractionated accelerated chemoradiotherapy in the conformal era achieved acceptable control rates for squamous cell carcinoma of the head and neck. This study reports outcomes for biologically staged oropharyngeal cancer treated using four-week intensity-modulated radiotherapy (IMRT) and synchronous chemotherapy. Between 2009-2012, patients with squamous cell carcinoma of the oropharynx treated with hypofractionated chemo-IMRT (55Gray in 20 fractions), with either carboplatin or cetuximab were prospectively identified. Outcome measures analysed were 2-year loco-regional recurrence-free survival (LR-RFS) and overall survival (OS). Eighty-five consecutive patients with oropharyngeal cancer ( $\mathrm{p} 16$ positive never smoked $\mathrm{n}=17$; p16 positive ever smoked $n=42$; p16 negative $n=24$; unknown $n=2)$ ) were treated with IMRT with carboplatin $(\mathrm{n}=69)$ or cetuximab $(\mathrm{n}=16)$, with median follow up in surviving patients of 26 months. Two year LR-RFS was $68 \%$ for the whole cohort (95\% confidence intervals 58\%-78\%), 87\% (58\%-97\%) for the p16 positive never-smokers, $75 \%(58 \%-86 \%)$ for the p16 positive ever-smokers and $45 \%(25 \%-63 \%)$ for the p16 negative patients. OS at 24 months for these groups respectively was $80 \%(69-87 \%), 100.0 \%, 90 \%(75 \%-96 \%)$ and $46.9 \%$ $(25 \%-66 \%)$. Factors associated with a worse OS on multivariate analysis were $\mathrm{p} 16$ negativity $(\mathrm{p}<0.001)$, age $>60$ $(\mathrm{p}=0.048)$, increasing $\mathrm{T}$ stage $(\mathrm{p}=0.021)$ and contraindication to carboplatin $(\mathrm{p}<0.001)$. The acceptable efficacy obtained with this schedule questions the need for synchronous cisplatin in good prognosis groups of oropharyngeal carcinoma. These results may also prompt the examination of a 4 week schedule of chemoradiotherapy in future randomised trials.
\end{abstract}

Keywords: acceleration, hypofractionation, oropharyngeal carcinoma, radiobiological modelling

\section{Introduction}

The use of a 4 week schedule of hypofractionated, accelerated radiotherapy for head and neck cancer was commonplace in the United Kingdom during the 1990's-2000's (James, Robertson, Squire, Jones, \& Cottie, 2003; Williams, James, Summers, Barrett, \& Ash, 2006; Tobias et al., 2009). This regimen has theoretical radiobiological advantages, as well as beneficial resource, cost and compliance implications over conventionally fractionated schedules (Fowler, 2007; Fowler, 2008). A 4 week regimen is currently being evaluated by the International Atomic Energy Agency (Overgaard et al., n.d.).

A number of series have since reported comparable efficacy and toxicity outcomes using this regimen with concurrent chemotherapy in patients with locally advanced squamous cell cancer of the head and neck (Tobias et al., 2009; Sykes, Slevin, MacDougall, Ironside, \& Mais; 2004, Sanghera, McConkey, Ho, Glaholm, \& Hartley, 2007; Jegannathan et al., 2010). More recently, an updated series by Chan et al. in 2011 reported acceptable local control outcomes and tolerability, with a 2 year local control of 78.3\% (Chan et al., 2011). However, patients in these series were treated with conformal radiotherapy, and so the applicability of results to patients treated with current standard of care, namely intensity-modulated radiotherapy (IMRT) utilising synchronous integrated boost, is unknown. 
Concurrent carboplatin chemotherapy has been used as an alternative to cisplatin in combination with hypofractionated accelerated radiotherapy. The safety of this regimen has been demonstrated in a phase I trial, and its efficacy and tolerability documented in a number of published series (Sanghera et al., 2007; Chan et al., 2011; Madhava, Hartley, Wake, Watkinson, \& Glaholm, 2006). Carboplatin offers potential benefits as an alternative to cisplatin with lower risks of systemic toxicity (in particular arterial thrombosis, nephrotoxicity and neurotoxicity), as well as a more convenient out-patient administration.

The epidermal growth factor receptor inhibitor Cetuximab used in combination with radiotherapy has been shown to be efficacious, and is approved by the United Kingdom National Institute for Clinical Excellence (NICE) as an alternative for patients with contra-indications to platinum chemotherapy with preserved performance status (Bonner et al., 2006, www.nice.org.uk/TA145).

p16 staining (as a surrogate marker for Human Papilloma Virus) and tobacco smoking status have been shown to be major independent prognostic factors (Ang et al., 2010). As a consequence, squamous cell carcinoma of the oropharynx can be staged biologically into risk groups based on p16 status and tobacco smoking history. All outcome data should now be stratified accordingly.

This study reports recent experience of hypofractionated accelerated IMRT with concurrent carboplatin or cetuximab in biologically staged patients with oropharyngeal cancer, and constitutes the largest series of such patients.

\section{Method}

\subsection{Data Collection}

Consecutive patients with biopsy-proven, non-metastatic squamous cell carcinoma of the oropharynx (International Union against Cancer [IUAC] stage II-IV) who received hypofractionated, accelerated IMRT with concurrent carboplatin or cetuximab between June 2009 and May 2012 at the Queen Elizabeth Hospital, Birmingham were identified.

Information collected from hospital notes included: patient characteristics; tumour sub-site and stage; p16 staining of tumour; smoking status; use of neo-adjuvant chemotherapy; pre-treatment haemoglobin level; total radiation dose; overall treatment time; details of any planned or salvage surgery; recurrence and survival data. Toxicity data were recorded in a database prospectively. Patients were assessed weekly with a clinical examination and blood profile (full blood count; renal and liver function) during, and after treatment until acute toxicities had resolved. Toxicity data including mucositis, skin reaction, nausea and dysphagia were graded using the National Cancer Institute common toxicity criteria version 3.0. Use of feeding tubes, length of opiate analgesia requirement and frequency and duration of hospital admissions were also recorded. Dependence on a feeding tube at 1 year and the incidence of prolonged confluent mucositis (Grade 3; 4 weeks after completion of chemoradiation) were recorded as late toxicity.

The main outcome measures studied were loco-regional recurrence-free survival and overall survival (OS).

\subsection{Radiotherapy}

All patients underwent computed tomography (CT) simulation with intravenous contrast, in a beam directional shell. CT simulation images were co-registered with a baseline gadolinium enhanced T1 weighted magnetic resonance imaging (MRI) scan if available. Gross tumour volume (GTV) was delineated using information from staging imaging (performed prior to neo-adjuvant chemotherapy if applicable), and clinical examination. The GTV was grown isotropically by 10 millimetres $(\mathrm{mm})$ and then enlarged further to include involved nodal levels, and edited to respect anatomical boundaries to form the high dose clinical target volume (CTV). This was extended to $15 \mathrm{~mm}$ in regions of uncertainty such as the base of tongue. At risk nodal levels that were both clinically and radiologically free of disease as well as the remaining oropharynx (not included in the high risk CTV) were delineated to form the low risk CTV. CTVs were expanded isotropically by $5 \mathrm{~mm}$ to form planning target volumes (PTV). The intended radiation dose to the high dose PTV was 55 Gray (Gy) in 20 fractions (2.75 Gy per fraction); treating 5 days a week over 4 weeks, with an overall treatment time (OTT) of 25 days. The intended dose to the low risk PTV was 46 Gy in 20 fractions (2.3 Gy per fraction). 50 Gy in 20 fractions was delivered to the neck in patients who had undergone a neck dissection. Standard organs at risk (OAR) were contoured with a $5 \mathrm{~mm}$ planning organ at risk volume (PRV) margin added to serial structures, including brainstem, cord, optic pathways and brachial plexus. An inverse planned IMRT optimisation was employed, with parotid sparing (where tumour coverage would not be compromised) and radiotherapy was delivered using either Tomotherapy ${ }^{\circledR}$ or 5-field step and shoot IMRT planned using either the Xio or Monaco treatment planning systems. 


\subsection{Chemotherapy}

The use of neo-adjuvant chemotherapy (docetaxel, cisplatin and 5-fluorouracil (TPF)) was reserved for patients with T4 and/or bulky nodal disease $>3 \mathrm{~cm}$, with no contra-indications. During the study period, two drug agents were used concurrently with radiotherapy. Patients either received outpatient carboplatin chemotherapy or cetuximab. The intended dose schedule for carboplatin was an area under the curve (AUC) dose of 4 in weeks 1 and 4. Cetuximab was given as an alternative to patients with a contra-indication to platinum based chemotherapy as per NICE guidelines, at a dose of 400 milligrams $(\mathrm{mg})$ per square metre $\left(\mathrm{m}^{2}\right) 1$ week prior to radiotherapy, followed by $250 \mathrm{mg} / \mathrm{m}^{2}$ weekly until completion of treatment.

\subsection{Statistical Analysis}

Overall survival (OS) and loco-regional recurrence-free survival (LR-RFS) at 2 years were analysed using Kaplan-Meier and log-rank methods. Proportional hazard models were used to test for effects of baseline characteristics, tumour and treatment variables on outcome measures. OS was defined from the start of radiotherapy until the date of last clinical review (patient censored at this point) or date of death if applicable. LR-RFS was calculated as the length of time from the start of radiotherapy to the date local or regional recurrence was diagnosed. Patients dying from intercurrent disease were censored at the time of death.

\section{Results}

\subsection{Patients}

Eighty-five consecutive patients with squamous cell carcinoma of the oropharynx received radical chemoradiotherapy (CRT) between June 2009 and May 2012. The median follow-up of surviving patients was 26 months. Baseline characteristics are summarised in Table 1.

Table 1. Baseline characteristics

\begin{tabular}{|c|c|c|}
\hline Characteristic & & \\
\hline Age, years. & Median (range) & $57(33-74)$ \\
\hline Sex, n (\%) & Male & $62(73 \%)$ \\
\hline & Female & $23(27 \%)$ \\
\hline Tumour subsite, n (\%) & Tonsil & $49(57 \%)$ \\
\hline & Base of Tongue & $33(39 \%)$ \\
\hline & Soft palate & $3(4 \%)$ \\
\hline IUAC Stage, n (\%) & I & $0(0 \%)$ \\
\hline & II & $3(4 \%)$ \\
\hline & III & $14(16 \%)$ \\
\hline & IV & $68(80 \%)$ \\
\hline Tumour stage, $\mathrm{n}(\%)$ & $\mathrm{T} 1$ & $14(16 \%)$ \\
\hline & $\mathrm{T} 2$ & $27(32 \%)$ \\
\hline & $\mathrm{T} 3$ & $15(18 \%)$ \\
\hline & $\mathrm{T} 4$ & $29(34 \%)$ \\
\hline Nodal stage, n (\%) & No & $9(11 \%)$ \\
\hline & N1 & $13(15 \%)$ \\
\hline & N2 & $59(69 \%)$ \\
\hline & N3 & $4(5 \%)$ \\
\hline Smoking status, n (\%) & Never smoked & $21(25 \%)$ \\
\hline & Former smoker & $33(39 \%)$ \\
\hline & Current smoker & $31(36 \%)$ \\
\hline Pre-radiotherapy haemoglobin level & $\leq 12.5$ & $43(51 \%)$ \\
\hline$(\mathrm{g} / \mathrm{dl}), \mathrm{n}(\%)$ & $>12.5$ & $42(49 \%)$ \\
\hline
\end{tabular}

(IUAC=International Union against Cancer, $\mathrm{g}=$ grams, $\mathrm{dl}=$ decilitre).

Tumour staining with p16 protein was analysable for $83(98 \%)$ patients. p16 positivity was defined by strong and diffuse nuclear and cytoplasmic staining in $70 \%$ or more of the tumour cells. Using this definition, 59 (71\%) tumours were positive for p16 expression, and 24 (29\%) were negative. Three groups were defined based on known prognostic variables: p16 positive never smokers; p16 positive ever smokers (current/ex) and p16 
negative patients regardless of smoking status. Distribution of patients between these groups is recorded in Table 2 .

Table 2. Prognostic groupings

\begin{tabular}{lc}
\hline Prognostic Group & \\
\hline $\begin{array}{l}\text { Good: } \\
\text { p16 positive, never smokers, n (\%) }\end{array}$ & $17(20 \%)$ \\
\hline $\begin{array}{l}\text { Intermediate } \\
\text { p16 positive, current/ex smokers, n (\%) }\end{array}$ & $42(51 \%)$ \\
\hline Poor: & $24(29 \%)$ \\
\hline 16 negative, $\mathrm{n}(\%)$ & \\
\hline
\end{tabular}

\subsection{Details of Treatment}

Of the 85 patients, 39 (46\%) received neo-adjuvant chemotherapy with docetaxel $75 \mathrm{mg} / \mathrm{m}^{2}$ (day 1 ), cisplatin 75 $\mathrm{mg} / \mathrm{m}^{2}$ (day 1) and 5-fluorouracil $750 \mathrm{mg} / \mathrm{m}^{2}$ (days 1-4) for 2-3 cycles. Eighty-three patients (98\%) received the intended radiation dose. Two patients chose to discontinue CRT before completing the intended schedule and declined further treatment. Both of these patients died as a result of locally progressive disease. Sixty-nine patients $(81 \%)$ received concurrent carboplatin chemotherapy, whilst $16(19 \%)$ had contra-indications to platinum based chemotherapy and were treated with weekly cetuximab.

Of the $76(89 \%)$ patients with regional nodal involvement, 23 (30\%) underwent a neck dissection; 14 before and 9 after CRT. Of these 9 patients who had neck dissection post CRT, 5 had planned surgery and the remaining 4 patients had post CRT imaging suspicious of persistent disease. Authors acknowledge the heterogeneity in use of neck dissection over this 3 year period. This was due to the gradual change in practice from planned neck dissection to imaging directed management, whilst the results of the PET-Neck trial are awaited.

\subsection{Treatment Toxicity}

Toxicity was recorded prospectively for the 83 patients who completed all planned treatment. Complete toxicity profiles were available for 75 patients in total. Grade 3 mucositis was experienced by all patients who completed the planned 4 weeks of radiotherapy. There was no grade 4 mucositis seen. Median duration of grade 3 mucositis was 28 days (range 7-65). Median duration of opiate use was 7 weeks, ranging from no use, to 102 weeks in a patient with locally recurrent disease. Grade 3 skin reaction was observed in 36 patients (48\%) with a median duration of 2 weeks (range 1-6). Patients receiving concurrent cetuximab were significantly more likely to experience grade 3 skin reactions versus those receiving carboplatin chemotherapy $(\mathrm{p}=0.002)$.

Thirty-six patients $(43 \%)$ had a percutaneous enteral gastrostomy (PEG) tube inserted prophylactically prior to starting CRT. Of the 47 patients $(57 \%)$ who did not have a prophylactic PEG tube inserted (who completed all planned treatment), 8 patients (17\%) required insertion of a nasogastric (NG) tube reactively during CRT. Thirty-seven patients (45\%) required non-elective admission to the ward during CRT for management of treatment related toxicity. Median duration of admission was 5 days (range 1-15). The principal reason for admission was for re-hydration, nutritional support and mucositis management in 30 patients $(81 \%)$, with the remaining patients being admitted for: treatment of infection ( 5 patients; $14 \%$ ), management of vomiting (1 patient; 3\%) and investigation of chest pain (1 patient; 3\%). Patients who did not have a PEG prior to the start of CRT were significantly more likely to be admitted to hospital during treatment versus those with a prophylactic feeding tube in place $(63 \%$ versus $22 \%$; $<<0.001)$.

Prolonged confluent mucositis, defined as persistent grade 3-4 mucositis 4 weeks after completion of CRT was present in $9(11 \%)$ patients. Late feeding dysfunction, i.e. dependence on a feeding tube at 1 year only occurred in 1 patient, who had also developed local recurrence.

\subsection{Tumour Outcome Measures}

Of the 83 patients who completed all planned treatment, 16 developed local recurrence ( 7 of whom also recurred in the regional nodes) after a median time of 10.5 months (range 3-21). Distant metastases developed in 5 patients (7\%), with a median time to diagnosis of 7 months (range 3-15). Of the 5 patients with distant metastases, none had recurrence in the neck or primary site. Four patients developed a second malignancy, all of which were lung in origin. Eighteen patients (21\%) have died, 14 as a result of their oropharyngeal malignancy, 
and 4 due to an unrelated cause. Two year loco-regional recurrence-free survival was $68 \%$ for the whole cohort (95\% confidence intervals $58 \%-78 \%), 87 \%(58 \%-97 \%)$ for the p16 positive never-smokers, $75 \%(58 \%-86 \%)$ for the p16 positive ever-smokers and $45 \%(25 \%-63 \%)$ for the p16 negative patients. Overall survival at 24 months for these groups respectively was $80 \%(69-87 \%), 100 \%, 90 \%(75 \%-96 \%)$ and $47 \%(25 \%-66 \%)$.

On multi-variate analysis, patients aged over 60 had a significantly worse 2 year OS than those $\leq 60(63 \%$ versus $88 \%$ respectively; $\mathrm{p}=0.048$ ). There was also a statistically significant association between increasing $\mathrm{T}$ stage of the tumour and 2 year LR-RFS $(\mathrm{p}<0.001)$ and OS $(\mathrm{p}=0.021)$. There were no significant differences in 2 year outcomes for patients stratified by nodal stage.

In this study, anaemia (defined as haemoglobin $\leq 12.5$ grams/decilitre) prior to commencement of CRT and the use of neo-adjuvant chemotherapy was not shown to significantly affect 2 year LR-RFS ( $p=0.85 ; p=0.096)$ or OS $(\mathrm{p}=0.57 ; \mathrm{p}=0.094)$.

There was a significant difference between patients with contra-indications to platinum based chemotherapy (implying treatment with concurrent cetuximab), and those who received carboplatin with regards to 2 year LR-RFS $(\mathrm{p}<0.001)$ and OS $(\mathrm{p}<0.001)$. Two year LR-RFS rates for patients receiving carboplatin versus cetuximab were $78 \%$ versus $31 \%$. Two year OS rates were $87 \%$ versus $50 \%$.

Patients with $\mathrm{p} 16$ positive oropharyngeal cancer had better LR-RFS and OS than those who did not $(\mathrm{p}=0.002$ and $\mathrm{p}<0.001$ respectively). Previous or current tobacco smoking when looked at independently did not demonstrate any significant relationship to 2 year outcome (either LR-RFS or OS). However, there were statistically significant differences between previously defined prognostic groups (combining p16 positivity and smoking status) and 2 year LR-RFS ( $\mathrm{p}=0.009)$ and OS $(\mathrm{p}<0.001)$. Table 3 summarises outcome data for these patient groups, and demonstrates statistically significant differences in OS and LR-RFS between them.

Table 3. Twenty-four month outcome data for whole cohort and patients defined by prognostic variables

\begin{tabular}{|c|c|c|}
\hline & $\begin{array}{l}\text { Loco-regional recurrence free survival, } \% \\
\qquad(95 \% \text { confidence interval })\end{array}$ & $\begin{array}{c}\text { Overall survival, } \%(95 \% \text { confidence } \\
\text { interval) }\end{array}$ \\
\hline & $\mathrm{p}=0.009$ & $\mathrm{p}<0.001$ \\
\hline Whole cohort & $68(58-78 \%)$ & $80(69-87 \%)$ \\
\hline P16 positive, never smokers & $87(58-97 \%)$ & 100 \\
\hline P16 positive, current/ex smokers & $75(58-86 \%)$ & $90(75-96 \%)$ \\
\hline P16 negative & $45(25-63 \%)$ & $47(25-66 \%)$ \\
\hline
\end{tabular}

\section{Discussion}

The present study forms the largest series to date of patients with biologically staged oropharyngeal cancer treated with a 4-week schedule of hypofractionated accelerated intensity-modulated chemoradiotherapy. Control rates for the whole cohort and patients classified by biological stage are equivalent to those in the series of patients treated in randomised trials reported by Ang et al.. Authors acknowledge that due to lack of prospectively collected smoking pack year data; prognostic groups used to classify outcomes in this study do not exactly match those used by Ang et al.. Although the local control of the whole cohort may appear lower than other recent UK series, unlike other series patients with contraindications to concomitant platinum have been included here (Chan et al., 2011; Loo, Geropantas, Wilson, Martin, \& Roques, 2013). For example, within the series by Loo et al., all patients received neoadjuvant TPF chemotherapy (Loo et al., 2013).

The authors acknowledge the limitations of retrospective, non-randomised data when interpreting these results. However, this study does raise a number of important questions. Firstly, it demonstrates the efficacy of a 4-week chemoradiation schedule, most notably in the most biologically favourable group of patients who in this series have a 2 year LR-RFS and OS of $87 \%$ and $100 \%$ respectively. Encouraging results in this biologically favourable group may warrant further evaluation within a phase 3 randomised trial comparing 4-week versus 7-week conventionally fractionated chemoradiation. Other trials are currently investigating de-intensification of treatment in this good prognosis p16 positive group. In particular, the ECOG E1308 phase II trial is using a reduced radiation dose in combination with cetuximab if patients achieve complete response at the primary site to neo-adjuvant chemotherapy (Marur et al., 2013). No outcome data is yet available. 
The use of synchronous carboplatin chemotherapy as an alternative to cisplatin in this study appears effective in the good prognosis group. The single randomised trial comparing conventionally fractionated radiotherapy alone versus cisplatin-radiotherapy and carboplatin-radiotherapy showed overall survival superiority for the cisplatin group (Fountzilas et al., 2004). However, this trial was relatively small and did not differentiate between patients based on prognostic variables which are now recognised. A recently published matched pair analysis comparing concurrent carboplatin versus cisplatin based CRT showed equivalence in local control and overall survival (Wilkins et al., 2013). Carboplatin, therefore, may be a valid option for good prognosis patients in an attempt to reduce toxicity.

NICE currently recommends the use of cetuximab with radiotherapy in patients with contra-indications to platinum based chemotherapy with preserved performance status (www.nice.org.uk/TA145). These are a different group of patients to those in the Bonner study, who were sufficiently fit to be randomised in a phase 3 trial (Bonner et al., 2006). In contrast, the use of cetuximab concurrently in our study was virtually synonymous with cardiovascular morbidity. Results from this study show that both LR-RFS and OS were significantly worse in the cetuximab group. This may suggest that cardiovascular morbidity is a significant prognostic factor in addition to p16 status and tobacco smoking. Although these results should not reflect on current studies evaluating the use of cetuximab in good prognosis groups (e.g. De-ESCALaTE HPV and RTOG 1016, it may call into question the efficacy of cetuximab in patients with co-morbid cardiovascular conditions and hence current NICE recommendations).

Certainly the role of non-chemotherapeutic synchronous agents in patients deemed unfit for cisplatin /carboplatin requires further investigation. In the UK the NIMRAD study (NIMorazole versus RADiotherapy alone) is about to open to recruitment to examine this question by randomising patients between radiotherapy alone and radiotherapy and nimorazole in patients who are not suitable for synchronous chemotherapy or cetuximab (http://clinicaltrials.gov/show/NCT01950689).

A possible benefit for the addition of nimorazole in the NIMRAD study is that hypoxia may be a more significant factor in patients deemed unsuitable for cisplatin due to vascular comorbidity. One of the concerns regarding hypofractionation and acceleration is that maximal reoxygenation may not occur during the shortened overall treatment time (Denekamp \& Joiner, 1982). The results in the good prognosis group suggest that this effect, if present, is minimal in this subgroup. For the whole population in this study, pre-treatment haemoglobin had no effect on prognosis in contrast to results of previous studies (Overgaard et al., 1989; Fein et al., 1995; Warde et al., 1998; Skladowski, Tarnawski, Maciejewski, Wygoda, \& Slosarek, 1999; van Acht, Hermans, Boks \& Leer, 1992; Dubray et al., 1996; Tamawski, Skladowski, \& Maciejewski, 1997). However, the local control of less than $50 \%$ in the poor prognosis sub-group certainly warrants further investigation of hypoxic sensitisation in this group.

Finally, the results of this study cast doubt on recent radiobiological modelling proposing that synchronous chemotherapy might act by suppression of accelerated repopulation (Meade et al., 2013; Meade, Mcconkey, Sanghera, Mehanna, \& Hartley, 2013). Two randomised trials comparing accelerated fractionation chemoradiation versus conventionally fractionated chemoradiation showed no additional benefit with acceleration (Ang et al., 2010; Bourhis et al., 2008). This prompted re-examination of the model proposing the conversion of the additional effect of chemotherapy into a radiation biologically effective dose (BED). However, the alternative model would suggest that the addition of synchronous chemotherapy to the radiation schedule used in this study would be associated with inferior efficacy outcomes when compared with conventionally fractionated chemoradiation. The biologically effective dose for tumour (tBED) using the model of Meade et al. $\left(\alpha=0.3 \mathrm{~Gy}^{-1} ; \alpha / \beta=10 \mathrm{~Gy} ; \mathrm{tp}=10\right.$ days; tk=22 days) would be $78 \mathrm{~Gy}_{10}$ for conventionally fractionated chemoradiation compared with only $69 \mathrm{~Gy}_{10}$ for the accelerated hypofractionated regimen used in this study (Meade et al., 2013). In this study this regimen does not appear obviously inferior to results with conventional fractionation. The alternative model of accounting for synchronous chemotherapy as additional BED $\left(\alpha=0.3 \mathrm{~Gy}^{-1}\right.$; $\alpha / \beta=10 \mathrm{~Gy} ; \mathrm{tp}=3$ days; $\mathrm{tk}=22$ days; $\mathrm{tBEDc}=9.3 \mathrm{~Gy}$ ) suggests near equivalence of these regimes with BEDs of $75 \mathrm{~Gy}_{10}$ and $77 \mathrm{~Gy}_{10}$ respectively. Alternatively, this data may support dose de-escalation for good prognosis groups. Further work is clearly required to define the optimum model for predicting outcomes following synchronous chemoradiation possibly modelling the effect of chemotherapy on the radiosensitivity parameter $\alpha$ and cell turnover time during radiation $\mathrm{Tp}$.

\section{Conclusion}

The acceptable efficacy obtained with this schedule, particularly in the good prognosis sub-group, may prompt the examination of hypofractionated accelerated schedules in future randomised trials. Similarly, the outcomes 
obtained with synchronous carboplatin question the need for concurrent cisplatin and its associated toxicity in the good prognosis sub-group. The appropriateness of the UK NICE guidance with respect to synchronous cetuximab in patients of good performance status but with contraindications to cisplatin needs further examination given the relatively poor outcome of such patients in this study. Finally, the radiobiological model of synchronous chemoradiation needs further revision to account both for the findings of this study and recent randomised trials of accelerated fractionation.

\section{References}

Ang, K. K., Harris, J., Wheeler, R., Weber, R., Rosenthal, D. I., Nguyen-Tân, P. F., .. Gillison, M. L. (2010). Human Papillomavirus and Survival of Patients with Oropharyngeal Cancer. $N$ Engl J Med, 363, 24-35. http://dx.doi.org/10.1056/NEJMoa0912217

Ang, K., Zhang, Q., Wheeler, R. H., Rosenthal, D. I., Nguyen-Tan, F., Kim, H. ... Weber, R. S. (2010). A phase III trial (RTOG 0129) of two radiation-cisplatin regimens for head and neck carcinomas (HNC): Impact of radiation and cisplatin intensity on outcome. J Clin Oncol, 28(15 Suppl), 5507.

Bonner, J. A., Harari, P. M., Giralt, J., Azarnia, N., Shin, D. M., Cohen, R. B., ... Ang, K. K. (2006). Radiotherapy plus cetuximab for squamous-cell carcinoma of the head and neck. $N$ Engl J Med, 354(6), 567-578. http://dx.doi.org/10.1056/NEJMoa053422

Bourhis, J., Sire, C., Lapeyre, M., Grégoire, V., Maingon, P., Calais, G., ... Auperin, A. (2008). Accelerated versus conventional radiotherapy with concomitant chemotherapy in locally advanced head and neck carcinomas: results of a phase III randomised trial (GORTEC 99-02). Int J Radiat Oncol Biol Phys, 72 : S31. http://dx.doi.org/10.1016/j.jijrobp.2008.06.836

Chan, A. K., Sanghera, P., Choo, B. A., McConkey, C., Mehanna, H., Parmar, S., ... Hartley, A. (2011). Hypofractionated Accelerated Radiotherapy with Concurrent Carboplatin for Locally Advanced Squamous Cell Carcinoma of the Head and Neck. Clin Oncol, 23, 34-39. http://dx.doi.org/10.1016/j.clon.2010.07.015

Denekamp, J., \& Joiner, M. C., (1982). The potential benefit from a perfect radiosensitizer and its dependence on reoxygenation. Br J Radiol, 657-663. http://dx.doi.org/10.1259/0007-1285-55-657-657

Dubray, B., Mosseri, V., Brunin, F., Jaulerry, C., Poncet, P., Rodriguez, J., ... Cosset, J. M. (1996). Anemia is associated with lower local-regional control and survival after radiation therapy for head and neck cancer: a prospective study. Radiology, 201, 553-558.

Fein, D. A., Lee, R., Hanlon, A. L., Ridge, J. A., Langer, C. J., CurranJr, W. J., ... Coia, L. R., (1995). Pretreatment hemoglobin level influences local control and survival of T1-T2 squamous cell carcinomas of the glottic larynx. Clin Oncol, 13, 2077-2083.

Fountzilas, G., Ciuleanu, E., Dafni, U., Plataniotis, G., Kalogera-Fountzila, A., Samantas, E. ... Ghilezan N. (2004). Concomitant radiochemotherapy vs radiotherapy alone in patients with head and neck cancer: a Hellenic Cooperative Oncology Group Phase III Study. Med Oncol, 21, 95-107. http://dx.doi.org/10.1385 /MO:21:2:095

Fowler, J. F. (2007). Is there an optimum overall time for head and neck radiotherapy? A review, with new modelling. Clin Oncol, 19, 8-22. http://dx.doi.org/10.1016/j.clon.2006.09.008

Fowler, J. F. (2008). Optimum overall times II: extended modelling for head and neck radiotherapy. Clin Oncol, 20, 113-126. http://dx.doi.org/10.1016/j.clon.2007.11.003

Fowler, J. F (2008). How much radiation is the chemotherapy worth in advanced head and neck cancer? .Int $J$ Radiat Oncol Biol Phys, 71, 326-329. http://dx.doi.org/10.1016/j.jirobp.2008.01.052

Hartley, A., Sanghera, P., Glaholm, J., Mehanna, H., McConkey, C., \& Fowler, J. (2010). Radiobiological modelling of the therapeutic radio for the addition of synchronous chemotherapy to radiotherapy in locally advanced squamous cell carcinoma of the head and neck. Clin Oncol, 22, 125-130. http://dx.doi.org/10.1016/j.clon.2009.10.004

James, N. D., Robertson, G., Squire, C. J., Jones. K., \& Cottier, B, (2003). A national audit of radiotherapy fractionation in head and neck cancer. Clin Oncol, 15, 41-46. http://dx.doi.org/10.1053/clon.2002.0198

Jegannathen, A., Swindell, R., Yap, B., Lee, L., Sykes, A., Mais, K., ... Slevin, N. (2010). Can synchronous chemotherapy be added to Accelerated Hypofractionated Radiotherapy in Patients with Base of Tongue Cancer? Clin Oncol, 22, 185-191. http://dx.doi.org/10.1016/j.clon.2009.12.007

Kasibhatla, M., Kirkpatrick, J., \& Brizel, D. (2007). How much radiation is the chemotherapy worth in advanced 
head and neck cancer? Int $J$ Radiat Oncol Biol Phys, 68, 1491-1495. http://dx.doi.org/10.1016/ j.ijrobp.2007.03.025

Lee, I., \& Eisbruch, A. (2009). Mucositis versus tumor control: the therapeutic index of adding chemotherapy to irradiation of head and neck cancer. Int J Radiat Oncol Biol Phys, 75, 1060-1063. http://dx.doi.org/10.1016/ j.ijrobp.2008.12.011

Loo, S. W., Geropantas, K., Wilson, P., Martin, W. M., \& Roques, T. W. (2013) Target-volume definition for intensity-modulated radiotherapy after induction chemotherapy and patterns of treatment failure after sequential chemoradiotherapy in locoregionally advanced oropharyngeal squamous cell carcinoma. Clin Oncol, 25(3), 162-170. http://dx.doi.org/10.1016/j.clon.2012.07.015

Madhava, K., Hartley, A., Wake, M., Watkinson, J. C., \& Glaholm, J. (2006). Carboplatin and hypofractionated accelerated radiotherapy: a dose escalation study of an outpatient chemoradiation schedule for squamous cell carcinoma of the head and neck. Clin Oncol, 18, 77-81. http://dx.doi.org/10.1016/j.clon.2005.08.014

Marur, S., Li, S., Cmelak, A., Gillison, M. L., Ferris, R. L., Bauman, J. E., Zhao, W., ... Burtness, B. (2013). E1308: A phase II trial of induction chemotherapy (IC) followed by cetuximab with low dose versus standard dose IMRT in patients with human papilloma virus (HPV)-associated resectable squamous cell carcinoma of the orophayrnx (OPSCC). JCO ASCO Annual Meeting, 31, Suppl, 6005. Retrieved from $\mathrm{http} / / /$ meetinglibrary.asco.org/content/111732-132

Meade, S., Sanghera, P., McConkey, C., Fowler, J., Fountzilas, G., Glaholm, J., ... Hartley, A. (2013). Revising the radiobiological model of synchronous chemotherapy in head-and-neck cancer: a new analysis examining reduced weighting of accelerated repopulation. Int $J$ Radiat Oncol Biol Phys, 86, 157-63. http://dx.doi.org/10.1016/j.ijrobp.2012.11.023

Meade, S., Mcconkey, C., Sanghera, P., Mehanna, H., \& Hartley, A. (2013). Revised radiobiological modelling of the contribution of synchronous chemotherapy to the rate of grades 3-4 mucositis in head and neck cancer. J Med Imaging Radiat Oncol, 57(6), 733-738. http://dx.doi.org/10.1111/1754-9485.12096

NICE technology appraisal guidance 145. (2008). Cetuximab for the treatment of locally advanced squamous cell cancer of the head and neck. Retrieved from http://www.nice.org.uk/TA145

NIMRAD. A Randomised Placebo-Controlled Trial of Synchronous NIMorazole versus RADiotherapy Alone in Patients with Locally Advanced Head and Neck Squamous Cell Carcinoma Not Suitable for Synchronous Chemotherapy or Cetuximab. Retrieved from http://clinicaltrials.gov/show/NCT01950689

Overgaard, J. (n.d.). International Atomic Energy Trial. Retrieved from http://nucleus.iaea.org/HHW/RadiationOncology/Radiation_Onclology_Research/CurrentIAEAresearch/C RP_E33035_H\%26N_HYPNO.pdf

Overgaard, J., Hansen, H. S., Andersen, A. P., Hjelm-Hansen, M., Jørgensen, K., Sandberg, E., ... Pederson, M. (1989). Misonidazole combined with split-course radiotherapy in the treatment of invasive carcinoma of larynx and pharynx: report from the DAHANCA 2 study. Int $J$ Radiat Oncol Biol Phys, 16, 1065-1068. http://dx.doi.org/10.1016/0360-3016(89)90917-6 xxxxx

Pettit, L., Meade, S., Sanghera, P., Glaholm, J., \& Hartley, A. (2014). Panoramic radiobiological modelling of the contribution of concomitant chemotherapy to biological effective dose in squamous cell carcinoma. Cancer and Clinical Oncology, 3(1).

Sanghera, P., McConkey, C., Ho, K. F., Glaholm, J., \& Hartley, A. (2007). Hypofractioned accelerated radiotherapy with concurrent chemotherapy for locally advanced squamous cell carcinoma of the head and neck. Int J Radiat Oncol Biol Phys, 67,1342-1351. http://dx.doi.org/10.1016/j.ijrobp.2006.11.015

Skladowski, K., Tarnawski, R., Maciejewski, B., Wygoda, A., \& Slosarek, K. (1999) Clinical radiobiology of glottic T1 squamous cell carcinoma. Int $J$ Radiat Oncol Biol Phys, 43, 101-106. http://dx.doi.org/10.1016/S0360-3016(98)00375-7

Sykes, A. J., Slevin, N. J., MacDougall, R. H., Ironside, J. A., \& Mais, K. L. (2004). Results of a phase I study to determine the maximum tolerated dose of capecitabine when given concurrently with radical radiotherapy in the treatment of squamous cell carcinoma of the head and neck. Radiother Oncol, 71(1),81-4. http://dx.doi.org/10.1016/j.radonc.2004.02.001

Tarnawski, R., Skladowski, K., \& Maciejewski, B. (1997). Prognostic value of hemoglobin concentration in radiotherapy for cancer of supraglottic larynx. Int $J$ Radiat Oncol Biol Phys, 38, 1007-1011. 
http://dx.doi.org/10.1016/S0360-3016(97)00308-8

Tobias, J. S., Monson, K., Gupta, N., MacDougall, H., Glaholm, J., Hutchison, I., ... Hackshaw, A. (2009) Chemoradiotherapy for locally advanced head and neck cancer: 10-year follow-up of the UK Head and Neck (UKHAN1) trial. Lancet Oncol, 10(9), 872-876.

Van Acht, M. J., Hermans, J., Boks, D. E., \& Leer, J. W. (1992). The prognostic value of hemoglobin and a decrease in hemoglobin during radiotherapy in laryngeal carcinoma. Radiother Oncol, 23, 229-235. http://dx.doi.org/10.1016/S0167-8140(92)80126-4

Warde, P., O'Sullivan, B., Bristow, R. G., Panzarella, T., Keane, T. J., Gullane, P. J., ... Cummings, B. J. (1998). T1/T2 glottic cancermanaged by external beam radiotherapy: the influence of pretreatment hemoglobin on local control. Int J Radiat Oncol Biol Phys, 41, 347-353. http://dx.doi.org/10.1016/S0360 -3016(98)00062-5

Wilkins, A. C., Rosenfelder, N., Schick, U., Gupta, S., Thway, K., Nutting, C. M., ... Bhide, S. A. (2013). Equivalence of cisplatin and carboplatin-based chemoradiation for locally advanced squamous cell carcinoma of the head and neck: a matched-pair analysis. Oral Oncol, 49(6), 615-619. http://dx.doi.org/10.1016/j.oraloncology.2013.02.004

Williams, M. V., James, N. D., Summers, E. T., Barrett, A., \& Ash, D. V. (2006). National survey of radiotherapyfractionation practice in 2003. Clin Oncol, 18, 3-14.

\section{Copyrights}

Copyright for this article is retained by the author(s), with first publication rights granted to the journal.

This is an open-access article distributed under the terms and conditions of the Creative Commons Attribution license (http://creativecommons.org/licenses/by/3.0/). 